\title{
Non-random aneuploidy specifies subgroups of pilocytic astrocytoma and correlates with older age
}

Adam M. Fontebasso ${ }^{1, *}$, Margret Shirinian'2,*, Dong-Anh Khuong-Quang ${ }^{3}$, Denise Bechet $^{3}$, Tenzin Gayden ${ }^{3}$, Marcel Kool ${ }^{4}$, Nicolas De Jay ${ }^{3}$, Karine Jacob ${ }^{3}$, Noha Gerges $^{3}$, Barbara Hutter ${ }^{5}$, Huriye Şeker-Cin ${ }^{4}$, Hendrik Witt ${ }^{4,6}$, Alexandre Montpetit ${ }^{7}$, Sébastien Brunet ${ }^{7}$, Pierre Lepage ${ }^{7}$, Geneviève Bourret ${ }^{7}$, Almos Klekner ${ }^{8}$, László Bognár ${ }^{8}$, Peter Hauser ${ }^{9}$, Miklós Garami ${ }^{9}$, Jean-Pierre Farmer ${ }^{10}$, Jose-Luis Montes ${ }^{10}$, Jeffrey Atkinson ${ }^{10}$, Sally Lambert ${ }^{11}$, Tony Kwan ${ }^{7}$, Andrey Korshunov ${ }^{12}$, Uri Tabori ${ }^{13}$, V. Peter Collins ${ }^{11}$, Steffen Albrecht ${ }^{14}$, Damien Faury ${ }^{3}$, Stefan M. Pfister ${ }^{4,6}$, Werner Paulus $^{15}$, Martin Hasselblatt ${ }^{15}$, David T.W. Jones ${ }^{4}$ and Nada Jabado ${ }^{1,3}$

${ }^{1}$ Division of Experimental Medicine, McGill University and McGill University Health Centre, Montreal, Quebec, Canada

2 Department of Experimental Pathology, Immunology and Microbiology, American University Of Beirut, Beirut, Lebanon

${ }^{3}$ Departments of Pediatrics and Human Genetics, McGill University and McGill University Health Centre, Montreal, Quebec, Canada

${ }^{4}$ Division of Pediatric Neurooncology, German Cancer Research Centre (DKFZ), Heidelberg, Germany

${ }^{5}$ Division of Theoretical Bioinformatics, German Cancer Research Centre (DKFZ), Heidelberg, Germany

${ }^{6}$ Department of Pediatric Oncology, Hematology and Immunology, University of Heidelberg, Heidelberg, Germany

${ }^{7}$ McGill University and Genome Quebec Innovation Centre, Montreal, Quebec, Canada

${ }^{8}$ Department of Neurosurgery, Medical and Health Science Center, University of Debrecen, Debrecen, Hungary

${ }^{9}$ 2nd Department of Paediatrics, Semmelweis University, Budapest, Hungary

${ }^{10}$ Department of Neurosurgery, Montreal Children's Hospital and McGill University Health Centre, Montreal, Canada

${ }^{11}$ Division of Molecular Histopathology, Department of Pathology, University of Cambridge, Cambridge, United Kingdom

${ }^{12}$ Clinical Cooperation Unit Neuropathology, German Cancer Research Center (DKFZ), Heidelberg, Germany

${ }^{13}$ Division of Pediatric Hematology-Oncology and The Labatt Brain Tumour Research Centre, The Hospital for Sick Children, Toronto, Canada

${ }^{14}$ Department of Pathology, Montreal Children's Hospital and McGill University Health Centre, Montreal, Canada

${ }^{15}$ Institute of Neuropathology, University Hospital Münster, Münster, Germany

*These authors have contributed equally to this work

Correspondence to: Nada Jabado, email: nada.jabado@mcgill.ca

David T.W. Jones, email: david.jones@dkfz-heidelberg.de

Keywords: pilocytic astrocytoma, aneuploidy, BRAF, MDM2, PLK2

Received: June 07, $2015 \quad$ Accepted: August 15, 2015 Published: September 10, 2015

This is an open-access article distributed under the terms of the Creative Commons Attribution License, which permits unrestricted use, distribution, and reproduction in any medium, provided the original author and source are credited.

\section{ABSTRACT}

Pilocytic astrocytoma (PA) is the most common brain tumor in children but is rare in adults, and hence poorly studied in this age group. We investigated 222 PA and report increased aneuploidy in older patients. Aneuploid genomes were identified in $45 \%$ of adult compared with $17 \%$ of pediatric PA. Gains were non-random, favoring chromosomes 5, 7, 6 and 11 in order of frequency, and preferentially affecting noncerebellar PA and tumors with BRAF V600E mutations and not with KIAA1549-BRAF fusions or FGFR1 mutations. Aneuploid PA differentially expressed genes involved in CNS development, the unfolded protein response, and regulators of genomic stability and the cell cycle (MDM2, PLK2), whose correlated programs were overexpressed specifically in aneuploid PA compared to other glial tumors. Thus, convergence of 


\section{pathways affecting the cell cycle and genomic stability may favor aneuploidy in PA, possibly representing an additional molecular driver in older patients with this brain tumor.}

\section{INTRODUCTION}

Pilocytic astrocytoma (PA) is a World Health Organization (WHO) grade I astrocytoma and the most common pediatric brain tumor $[1,2]$. These tumors generally have an excellent prognosis and limited morbidity when amenable to gross total surgical resection $[2,3]$, with the highest age-adjusted incidence rate occurring in children between $0-19$ years old when assessed across the lifespan [4]. In children, PAs most commonly occur in the cerebellum, and with decreasing frequency in other more surgically challenging brain locations including the optic pathway, thalamus, brainstem, spinal cord and cerebral hemispheres [5]. In adults, the reverse gradient for location is observed, and the low incidence of PA largely contributes to the relative lack of molecular investigations in this setting [5].

Recent genomic studies have shown that, in children, virtually all PAs can be categorized as harboring an alteration leading to constitutive activation of the MAPK pathway. The most common genetic alteration $(\sim 65 \%)$ is a tandem duplication at $7 \mathrm{q} 34$ creating an inframe fusion gene between BRAF and KIAA1549 [6-9]. Other alterations of the pathway include $R A F$-family fusions [7, 10], BRAF V600E mutation [11], germline $N F 1$ alterations (reviewed in [8]), and fibroblast growth factor receptor $1(F G F R 1)$ and NTRK-family alterations $[9,12]$. In contrast, lower frequencies of $R A F$ fusion transcripts have been reported in adult PAs [13]. Importantly, 50$60 \%$ of PA in patients over the age of 20 years lacked a KIAA1549:BRAF fusion, BRAF V600E mutation or FGFR1 mutation [14]. This suggests that these tumors may harbor some of the rarer MAPK pathway alterations, or that other putative driver alterations or programs may additionally contribute to the molecular landscape of adult PA.

Whole-chromosomal copy number gains have been reported in a small cohort of 44 PA analyzed using array-based comparative genomic hybridization (aCGH) and seemed to favor older patients [15]. The prevalence, relation to patient age and significance of this phenotype as well as its relation to $B R A F$ and MAPK alterations and possible underlying mechanisms remain, however, largely uncharacterized. As such, we analyzed a large combined dataset of 222 adult and pediatric PAs with wholechromosomal copy number data, including tumors from previously published aCGH and SNP array studies $[6,7$, $15-17]$. We show that aneuploidy is a common feature of the genomes of adult PA, affecting $45 \%$ of tumors in this age range. This is a non-random process that favors gains but not losses, specifically in chromosomes 5, 7, 6 and 11. It is strongly associated to non-cerebellar regions, which are less amenable to gross-total surgical resection, and to tumors harboring $B R A F$ V600E mutation, which may be one of the molecular drivers of this phenotype. Pathways favoring aneuploidy are differentially regulated in adult PA, with many expression changes mapping to chromosome 5. This includes central nervous system (CNS) developmental pathways, unfolded protein response pathways and the cell cycle, including overexpression of $M D M 2$ and $P L K 2$, and specific gene expression signatures we identify to be strongly correlated with aneuploid PA. Furthermore, our findings suggest a link between aging and the physiological non-random aneuploidy which occurs in the brain and may predispose to this brain tumor.

\section{RESULTS}

\section{Non-random aneuploidy characterizes adult PA tumors arising throughout the CNS}

We combined published datasets of wholechromosomal copy number analyses of PA tumors $[6,7,15-17]$ with newly-derived profiles from DNA methylation data [10] as well as newly-analyzed tumor samples, and assembled copy number data for a combined series of 222 PAs ( $n=44$ adult; $n=178$ pediatric). In our combined dataset, whole chromosomal gain in at least one chromosome was common, occurring in $22.5 \%$ (50/222) tumors overall (Figure 1a-1d; Supplementary Table 1). This aneuploidy was present in PA tumors occurring throughout the central nervous system (CNS), with a notable fraction occurring in surgically challenging areas such as the thalamus, hypothalamus, optic pathway, brainstem, 4th ventricle and spinal cord (Figure 1b). Aneuploidy tended to favor tumors occurring in non-cerebellar areas when compared with the location distribution of the euploid cohort, a trend which approached statistical significance $(P=0.0552$; Fisher's Exact Test). Aneuploidy was significantly more common in the adult PA subset $(20 / 44,45 \%$ vs. $30 / 178$, $17 \% ; \mathrm{P}=0.0002$; Fisher's Exact Test) (Figure 1c) with the average age at diagnosis of patients with aneuploid PA tumors being about 10 years older than those with euploid tumors $(19.5+/-2.1$ years, compared to $9.9+/$ 0.8 years, respectively; $P<0.0001$; unpaired, twotailed t-test) (Figure 1d). The frequency of individual chromosomes affected demonstrated a specific pattern across the 50 aneuploid PAs, with the most commonly altered chromosomes in order of frequency being chromosomes 5, 7,6 and 11, in keeping with previous findings [15]. These four chromosomes were statistically over-represented in overall events among the aneuploid tumors $(P<0.0001)$. In addition, chromosomes $1,2,3,13$, 
$1416,17,19$ and 22 were significantly under-represented across the background of all changes $(P<0.05)$ (Figure 1a). Aneuploidy did not appear to correlate with different progression-free survival (PFS) profiles through KaplanMeier analysis (Supplementary Figure 3).

\section{Aneuploidy and MAPK alterations in PA}

By integrating aneuploidy and BRAF fusion status, we determined that tumors wild-type for $B R A F$ fusion demonstrated an increased rate of aneuploidy $(36 \%, 23 / 64$ vs $18 \%, 27 / 150 ; P=0.0076$; Fisher's Exact Test) (Figure 2a). Conversely, an increased frequency of aneuploidy is seen in tumors harboring BRAF mutations (53\%, 8/15 vs 20\%,32/127; $P=0.0074$; Fisher's exact test) (Figure 2b). Aneuploidy did not correlate significantly with FGFR1 mutation status $(P=0.68$; Fisher's Exact Test). Using
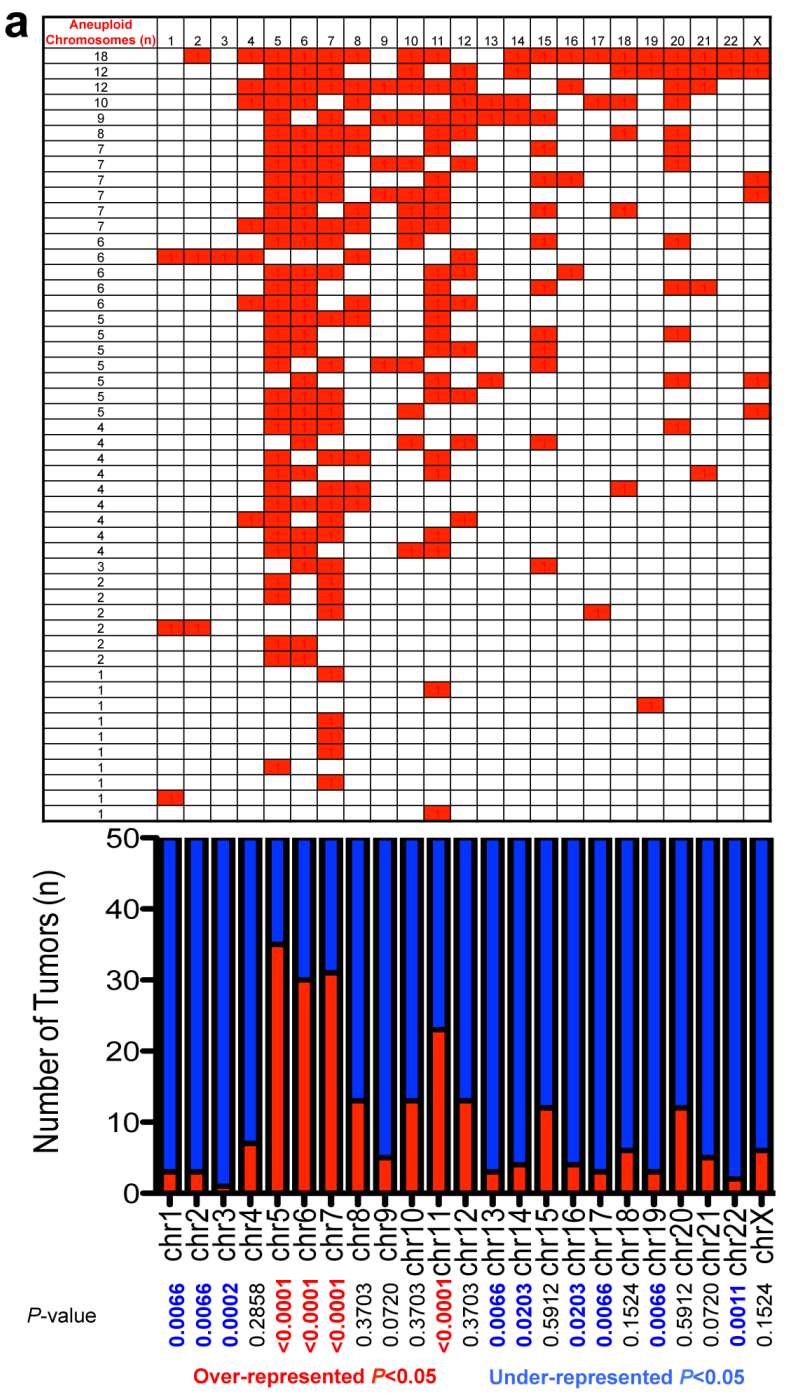

the R2 microarray analysis and visualization platform (http://r2.amc.nl), we identified differential expression of 558 genes (FDR $<0.001$, ANOVA) between euploid ( $n$ $=93$ ) and aneuploid tumors $(n=29)$ (Figure 2c). Other comparisons demonstrated fewer genes differentially regulated at FDR $<0.001$, including BRAF fusion positive vs negative (29 genes), BRAF mutation positive vs negative (216 genes) and age group categories (462 genes), demonstrating a strong effect of aneuploidy on global gene expression patterns in PA (Figure 2c).

\section{Pathways aberrantly regulated in aneuploid tumors}

Gene Ontology (GO) analysis of the 558 genes differentially regulated between euploid and aneuploid tumors revealed significance of molecular pathways
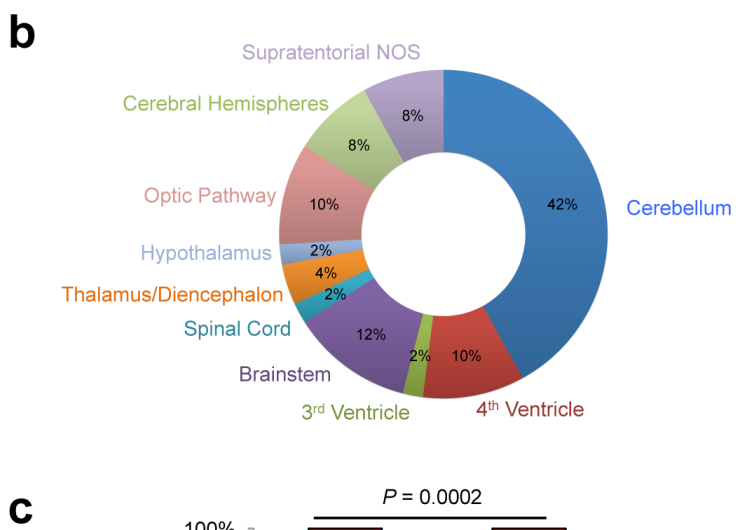

C

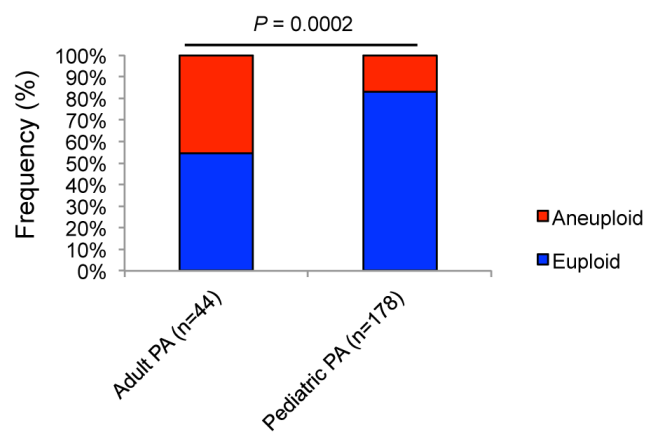

d

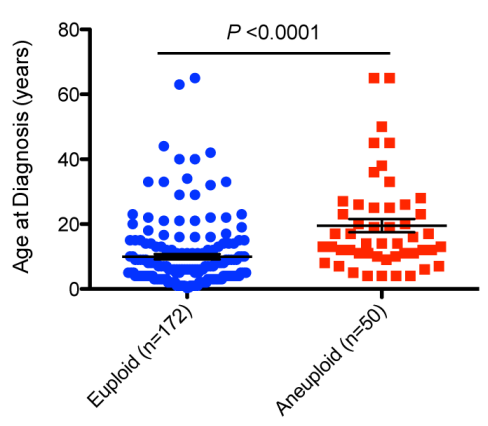

Figure 1: Characterization of aneuploidy observed in pilocytic astrocytoma. a., Gains in 50 aneuploid PA samples (upper panel), frequency of specific gains, and significance $(P<0.05$; Fisher's Exact Test; lower panel). b., Neuroanatomical distribution of aneuploid PA tumors favors non-cerebellar areas. c., Increased aneuploidy observed amongst adult PA tumors $(P=0.0002$; Fisher's Exact Test). d., Age of patients with aneuploid and euploid PA tumors $(P<0.0001$; unpaired two-tailed $t$-test). 
including GO terms such as CNS development (GO: 7417), cell cycle arrest (GO: 7050), G1/S transition of the mitotic cell cycle (GO: 82) and unfolded protein binding (GO: 51082) (Figure 3a). Mapping of these 558 genes to their respective chromosomal locations revealed enrichment (over-representation of differentially expressed genes) on chromosomes 5 and 7, and underrepresentation of chromosomes 1, 10, 14, 17 and 19 (Figure $3 \mathrm{~b}$ ), mirroring the relative frequency of gains of these chromosomes seen in our cohort. Candidate genes significantly de-regulated between aneuploid and euploid PA tumors included MDM2 (Figure 3c), encoding the E3 ubiquitin ligase MDM2, and polo-like kinase 2 (PLK2) (Figure 3d). These genes were also strongly correlated with each other in the 122 PA tumors with available gene expression and ploidy status $(P=0.01$, Figure $3 \mathrm{e})$.

\section{MDM2 is highly expressed in aneuploid PA}

Utilizing the R2 database tool, we sought to investigate $M D M 2$ expression comparatively across PA, as well as in other gliomas and control brain samples.

\section{a}
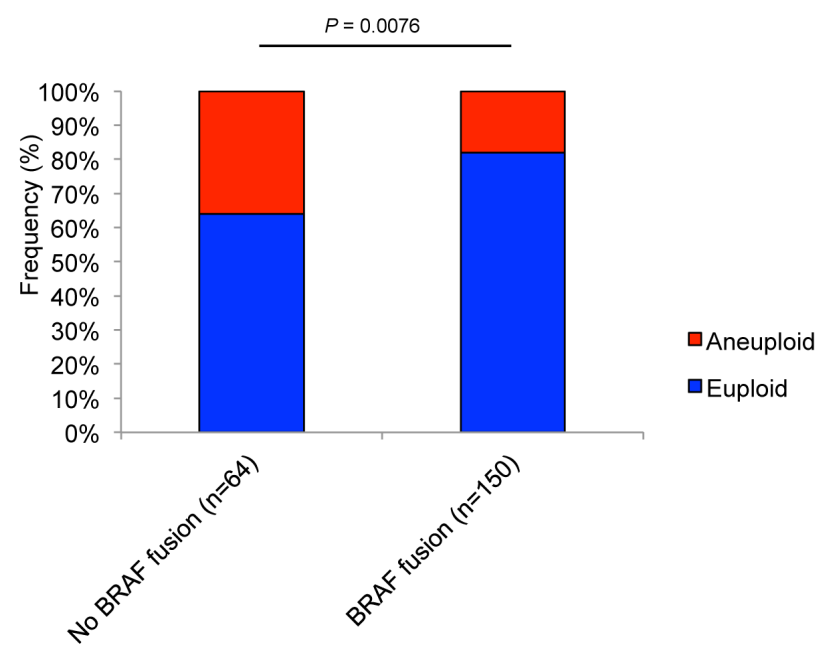

Across multiple gene expression datasets including PA, adult glioblastoma (GBM), pediatric high-grade glioma (pHGG), diffuse intrinsic pontine glioma (DIPG), ependymoma (EP) and normal brain (NB) and cerebellum (CB) samples, aneuploid PA tumors demonstrated the highest levels of $M D M 2$ expression (Figure 4a). Further dissection of $M D M 2$ expression within the PA dataset with available clinical and molecular variables revealed a strong association of $M D M 2$ over-expression in adult PA samples $\left(P=1.1 \times 10^{-4}\right.$; ANOVA) (Figure $\left.4 \mathrm{~b}\right)$, tumors wild-type for $B R A F$ fusion $\left(P=8.0 \times 10^{-6}\right.$ ANOVA) (Figure $\left.4 \mathrm{c}\right)$ and tumors harboring $B R A F$ mutations $\left(P=9.3 \times 10^{-7}\right.$; ANOVA) (Figure $4 \mathrm{~d}$ ), mirroring our findings as to the association of these factors with aneuploidy. Even slight elevations in $M D M 2$ expression have been reported to accelerate tumorigenesis (e.g. in association with the MDM2 SNP 309 [18, 19]). Manual curation of DNA copy number profiles revealed PA genomes largely deficient in focal copy number alterations other than $B R A F$ duplication and whole-chromosomal aneuploidy, suggesting mechanisms other than MDM2 genomic amplification as causal for $M D M 2$ overexpression (data not shown). Consequently, we investigated whether this MDM2 over-expression is

\section{b}

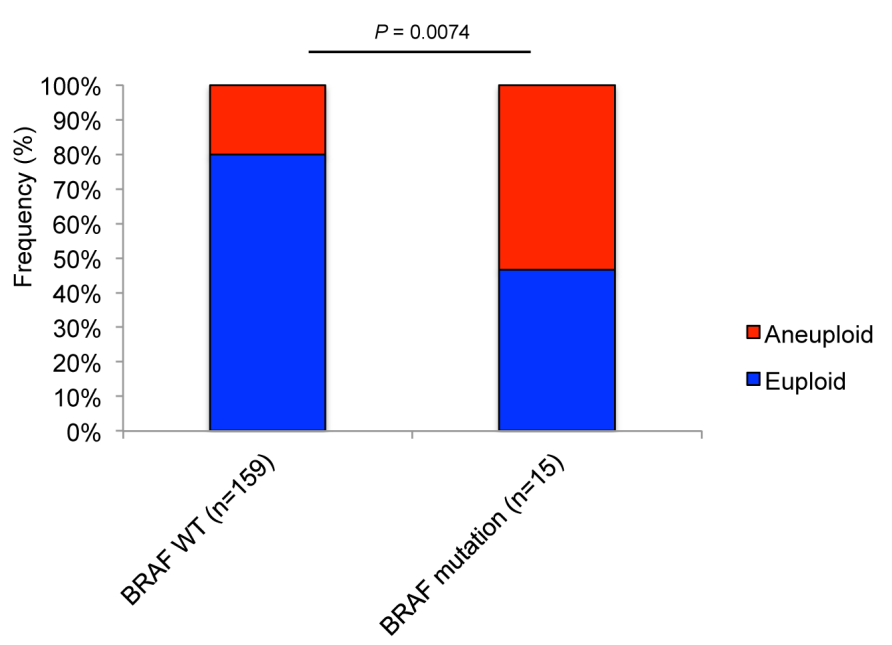

C

\begin{tabular}{|lcc|}
\hline Category & $\begin{array}{c}\text { Number of } \\
\text { Genes } \\
\text { (FDR<0.001) }\end{array}$ & $\begin{array}{c}\text { Tumors with } \\
\text { Data Available } \\
\text { (n) }\end{array}$ \\
\hline Aneuploidy & 558 & 122 \\
\hline BRAF Fusion & 29 & 124 \\
\hline BRAF Mutation & 216 & 108 \\
\hline Age Group & 462 & 126 \\
\hline
\end{tabular}

Figure 2: Aneuploidy is differentially associated with prominent MAPK alterations in PA with a strong effect on global gene expression. a., Frequency of aneuploidy within $B R A F$ fusion $(P=0.0076$; Fisher's Exact Test $)$ a. and $B R A F$ mutation $(P=0.0074$; Fisher's Exact Test) b. subsets of PA tumors. c., Strong effect of aneuploidy on global gene expression in PA at FDR $<0.001$. 
associated with the well-characterized promoter SNP 309 of $M D M 2$, which influences expression from the P2 promoter of MDM2 (Supplementary Figure 1a), we sequenced this SNP in 201 tumors and looked for correlations with $M D M 2$ expression and ploidy status where available (Supplementary Table 1, Supplementary Figure 1b-1e). SNP 309 TT, TG and GG genotypes were differentially associated with aneuploidy, although this did not reach statistical significance $(P=0.0926$; Chi-square test). SNP 309 status also did not consistently correlate with overall levels of MDM2 expression either by arraybased analysis or in a small validation cohort of tumor RNA samples using qPCR (Supplementary Figure 1c1d). No differences of MDM2 P1 and P2 promoter usage, which is affected by SNP 309 (reviewed in [20]), were observed in exon-level RNA-sequencing analysis using a previously reported dataset [9] (Supplementary Figure 1e), suggesting other mechanisms upregulate MDM2 in aneuploid PA tumors (Supplementary Figure 1a).

\section{$M D M 2$-correlated gene signatures are robust in pilocytic astrocytoma}

Utilizing the R2 database tool, we analyzed genome-wide gene expression correlations with $M D M 2$ (absolute correlation, FDR $<0.001$ ) in PA and other gliomas to determine candidate programs that may lead to aneuploidy in these tumors. Several hundred genes correlated very strongly with the expression of MDM2 within a PA dataset of 122 tumors profiled for ploidy status (741 genes at FDR $<0.001)$. Interestingly, in other publicly available glioma datasets including pediatric high-grade gliomas [21], DIPGs [22] and ependymomas [23], such correlations with MDM2 expression do not exist, with very few genes passing the same false discovery rate criteria (FDR $<0.001)$ although this might be partially explained by PAs being very homogeneous entities whereas other tumor types are mixtures of multiple subgroups (Supplementary Figure

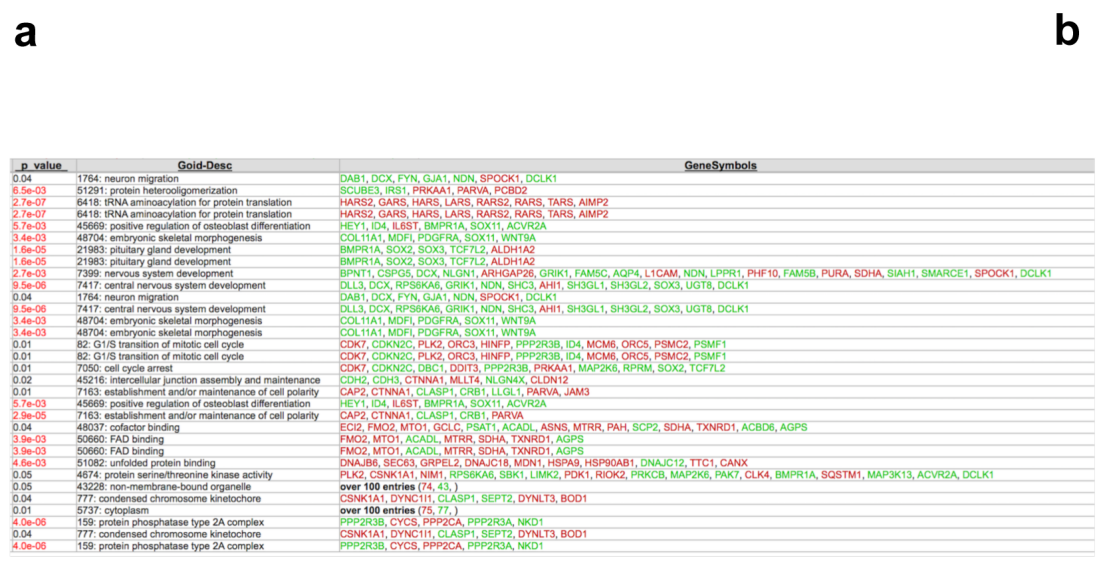

b

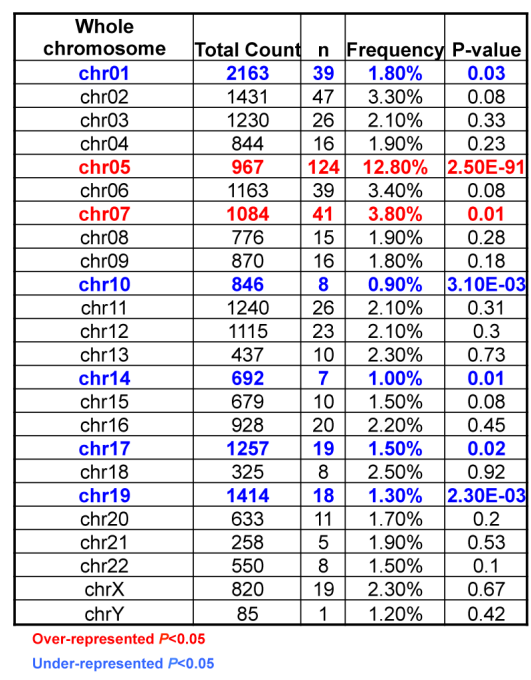

C

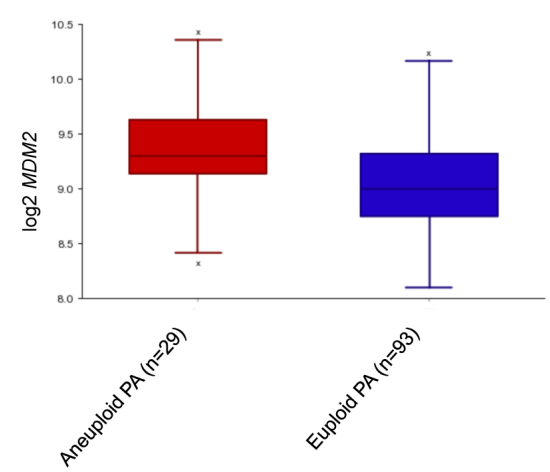

d

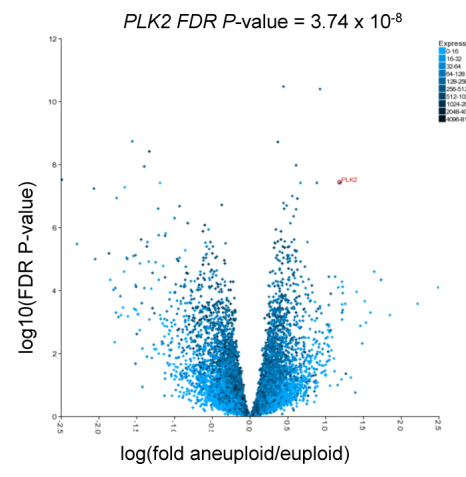

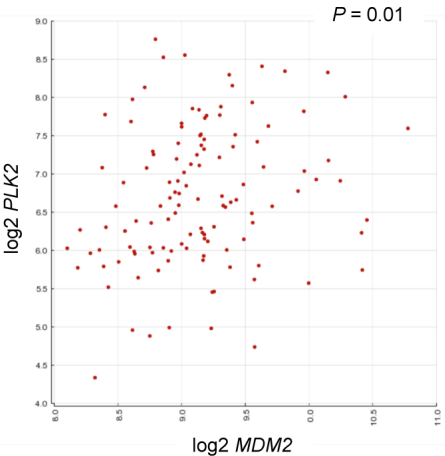

$\log 2 M D M 2$

Figure 3: Comparative gene expression analysis identifies specific pathways aberrantly regulated in aneuploid tumors. a., Aneuploidy signatures demonstrate enrichment of CNS development, cell cycle and unfolded protein binding pathways. b., Chromosomal mapping of the 558 genes demonstrates enrichment within chromosomes involved in aneuploid gains. c., d., Over-expression of candidates $M D M 2$ c. and $P L K 2$ (d) within the aneuploid PA subgroup $\left(P=1.5 \times 10^{-3}\right.$ and FDR adjusted $P$-value $=3.74 \times 10^{-8}$ respectively; ANOVA $)$. e., Correlation of $M D M 2$ and $P L K 2$ expression in 122 PAs with available aneuploid data $(P=0.01)$. 
2a-2c). Gene Ontology (GO) analysis (Supplementary Table 2) revealed significant GO terms corresponding to protein ubiquitination during ubiquitin-dependent protein catabolic process (GO: 42787), DNA damage response, signal transduction by p53 class mediator resulting in cell cycle arrest (GO: 6977), CNS development (GO: 7417), ligase activity (GO: 16874), cell cycle arrest (GO: 7050), cell cycle checkpoint (GO: 75) and G1 phase of mitotic cell cycle (GO: 80) amongst others (Supplementary Table 2). Utilizing the R2 chromosome mapping tool we mapped the 741-gene signature to respective chromosomal positions to determine any specific enrichment across the genome. In contrast to the aneuploidy signature, correlated genes appeared to be over-represented across chromosome $19\left(P=4.0 \times 10^{-5}\right)$ and chromosome $12\left(P=7.5 \times 10^{-3}\right)$, but not any other chromosome.

\section{BRAF-fused PA tumors comprise molecular entities with distinct characteristics}

We analyzed various clinical and molecular characteristics of tumors with $B R A F$ fusions compared to tumors without $B R A F$ duplications/fusions (Supplementary Table 3). Tumors harbouring $B R A F$ fusions/duplications were much more likely to be located in the infratentorial compartment $(P<0.0001)$, to be wild-type for $F G F R 1 / B R A F$ mutations $(P<0.0001)$, to be euploid $(P=0.0076)$ and to occur in children of younger mean age (11.1 years compared to 19.8 years). No statistically significant difference in progressionfree survival was observed between patients with tumors positive or negative for $B R A F$ duplication/fusion (Supplementary Figure 3, Supplementary Table 3), in keeping with data observed previously in adult PA patients [24].

\section{a}
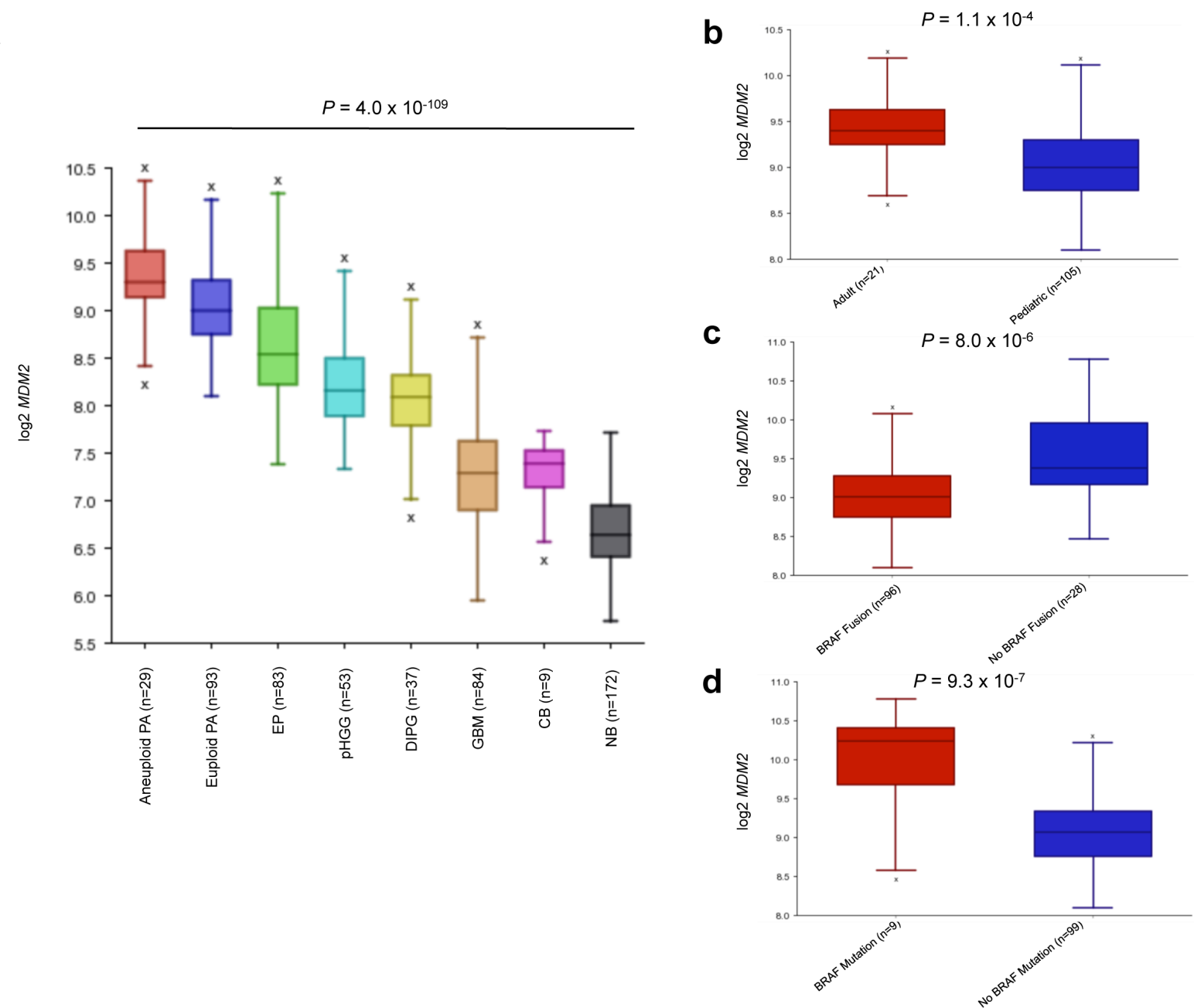

Figure 4: MDM2 expression analysis of CNS tumors and tissues reveals high expression in aneuploid PA. a., $M D M 2$ expression is highest in aneuploid PA tumors $\left(P=4.0 \times 10^{-109}\right.$, ANOVA $) . \mathrm{NB}=$ normal brain, $\mathrm{CB}=$ normal cerebellum, $\mathrm{GBM}=$ glioblastoma, $\mathrm{DIPG}=$ diffuse intrinsic pontine glioma, $\mathrm{pHGG}=$ pediatric high-grade glioma, $\mathrm{EP}=$ ependymoma, $\mathrm{PA}=$ pilocytic astrocytoma. $\mathbf{b}$., $\mathbf{c}$., d., $M D M 2$ over-expression in adult patients $(>18$ years $)\left(P=1.1 \times 10^{-4}\right)$ b., $B R A F$ fusion negative $\left(P=8.0 \times 10^{-6}\right)$ c. and $B R A F$ mutant $P A(P=$ $\left.9.3 \times 10^{-7}\right)$ d.. $P$-values calculated using ANOVA. 


\section{DISCUSSION}

We identify herein in a large cohort of 222 adult and pediatric PA located throughout the CNS, non-random chromosomal gains with a substantial enrichment of chromosomes 5, 6, 7 and 11 . This aneuploidy genotype predominates in older patients with PA, in keeping with an earlier aCGH report on $44 \mathrm{PA}$ samples [15]; mainly occurs in extra-cerebellar locations, and favors tumors that carry $B R A F$ V600E but not BRAF fusions or FGFR1 mutations. Aneuploid tumors had increased levels of $M D M 2$ and $P L K 2$ which are involved in cell-cycle arrest and ploidy control and may contribute to the observed genotype. Importantly, aneuploidy was identified in a large number of older patients with PA, and may represent an additional molecular driver in these samples.

Whole-chromosome alterations have been recurrently observed in several cancer types including mainly leukemias and sarcomas $[25,26]$. Aneuploidy in brain tumors is considerably less well understood and has been under-explored. In normal brain, aneuploidy has been reported as a phenomenon of the aging mammalian CNS (reviewed in [27]). In the developing mouse brain, it affects various chromosomes in 33\% of embryonic cortical [28] or postnatal subventricular zone [29] neuroblasts. Notably, aneuploidy is a common feature of the developed CNS [30] and displays a predilection to older mice [31], glial cells [31], specific chromosomes (murine chromosomes 7, 18 and Y), and the brain cortex [31], which shows significant increased age-related aneuploidy compared to the cerebellum (which remains euploid). These observations are consistent with our findings that aneuploid tumors are enriched in older patients and noncerebellar areas, where $B R A F$ fusions have the lowest incidence. However, we cannot formally exclude that some of the genomic alterations we see in adult PAs are not due to tumor environment differences associated with age, brain maturation and endocrine context. $[8,14]$.

BRAF expression and signaling is required for normal development of the cerebellum and hindbrain structures, and consequently cellular and murine studies of the KIAA1549-BRAF fusion show preferential growth selectivity in cerebellar neural stem cells $[32,33]$. Within our dataset, aneuploid PA had significantly decreased frequency of $B R A F$ fusion and mainly occurred outside cerebellar and hindbrain structures. They were, however,
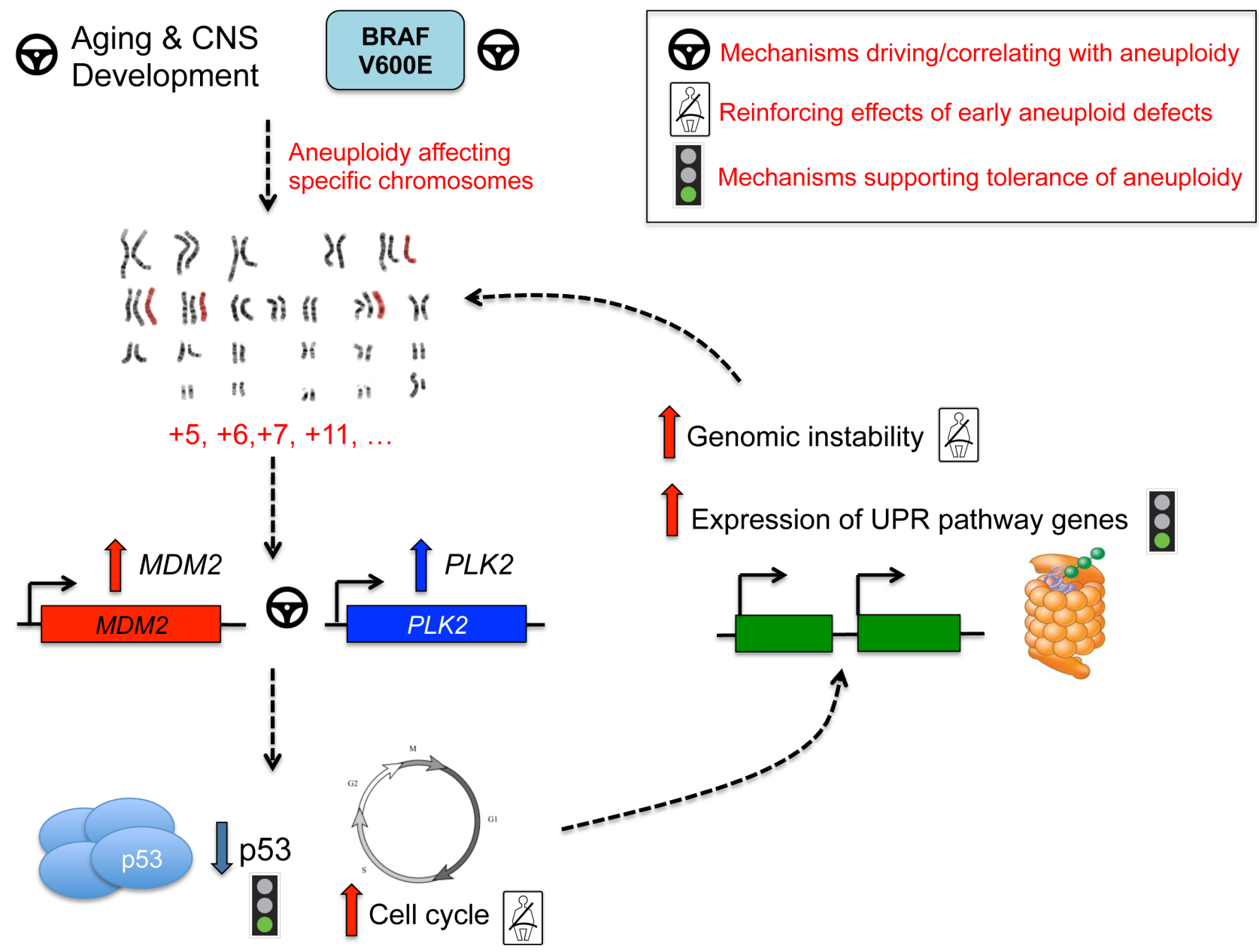

Figure 5: Pathway convergence leading to aneuploidy in PA. 
associated with higher frequencies of $B R A F \mathrm{~V} 600 \mathrm{E}$, a genetic alteration enriched in PA located in extra-cerebellar regions. Interestingly, this mutation has been shown to induce aneuploidy through spindle anomalies and supernumerary centrosomes in melanocytic and melanoma cells [34-36]. Whether these opposing associations of $B R A F$ fusion or activating mutation with aneuploidy in PAs is driven by subcellular expression patterns, brainlocation specific differences or MAPK-independent functions based on brain regions will necessitate future mechanistic studies.

Aberrant regulation of developmental pathways may point to unique cellular origins of aneuploid PA tumor cells, or to developmental programs being reactivated from inherent early mammalian developmental stages which demonstrate aneuploidy and are proposed to underlie cellular diversity of the CNS (reviewed in[27]). Significance of pathways involved in the unfolded protein response and protein ubiquitination potentially illustrates that aneuploid PA tumors harbor mechanisms to tolerate stress accompanying extra chromosomal content. Indeed, chromosomal gains are accompanied by substantial cellular stress of increased gene and protein expression[37] and studies in yeast demonstrate that aneuploidy causes proteotoxic stress, including accumulation of proteins more challenging to fold[38]. Aneuploidy may thus represent a large-scale "stress-inducible mutation"[39]. The triggering of the unfolded protein binding pathways may thus represent a mechanism that aneuploid tumors use to adapt and permit unadulterated growth of aneuploid cells (Figure 5). Alterations in the cell cycle can contribute to mitotic checkpoint dysregulation, continued cell growth and ultimately aneuploid defects[40]. Polo-like kinases, including PLK1 and PLK2, are critical regulators of cell division[41-43] and PLK2 is required for proper centriole duplication [42-47]. In our dataset, PLK2 was over-expressed in aneuploid PA tumors, and presents an interesting candidate for promotion and reinforcement of aneuploidy. Many genes differentially regulated between aneuploid and euploid tumors in our dataset mapped to commonly trisomic chromosomes, with striking enrichment seen across chromosome $5\left(P=2.50 \times 10^{-91}\right)$. With such high frequency, chromosome 5 gains are not only well-tolerated in PA tumors, but may actively reinforce aneuploid phenotypes based on candidate mapping of genes including PLK2 (Figure 5). MDM2 overexpression was also significantly associated with aneuploid PA and older patients. It strongly correlated with $P L K 2$ expression. $M d m 2$ transgenic mice have increased age-related aneuploid defects affecting the rate of chromosome gain, but not loss [48, 49] similar to what is seen in our PA dataset. MDM2 targets p53 for degradation and its amplification/overexpression mimics TP53 loss[50, 51]. The p53 regulatory pathways plays a critical role in limiting the progression of aneuploidy and preserving the nature of diploid human cells [52]. There are no reported
TP53 alterations in PA $[9,12]$, but increased MDM2 expression in aneuploid PA tumors may underlie inhibition of p53 function and subsequent continued growth of aneuploid cells. Our data show that a statistically robust 741-gene signature, correlated with MDM2 expression, exists in a large dataset of 122 PA tumors, but not other pediatric gliomas. GO analysis of these $M D M 2$ correlated genes also points to cyclin-dependent kinases, cell cycle arrest and checkpoint pathways, ligase activity and CNS development (Supplementary Table 2). This data suggests a central role for $M D M 2$ mediated programs that may cause and/or tolerate aneuploidy in PA (reviewed in[40]) (Figure 5). Moreover, maintenance of aberrant p53 signaling through $P L K 2$ has been identified in previous studies[53], further suggesting an intriguing link between PLK2 and MDM2 overexpression and defective p53 cell cycle control that may further promote aneuploidy and tumor formation. Future functional studies in the context of mediating aneuploidy in PA are needed to validate these concepts.

In summary, with the involvement of potential driving, reinforcing and tolerating mechanisms of aneuploidy, we describe intriguing targets for both future mechanistic and diagnostic/therapeutic study of this phenotype and its role in (adult) PA tumorigenesis. As these adult PA show a reduced incidence of the most common genetic alterations associated with pediatric PA, this non-random aneuploidy may be an additional genetic modifier in this setting, and is potentially triggered by aging mechanisms in glial cells of the brain and further promoted by the molecular alterations we identify herein.

\section{MATERIALS AND METHODS}

\section{Sample characteristics and pathological review}

All samples were obtained with informed consent after approval of the Institutional Review Board of the respective hospitals they were treated in, and independently reviewed by senior pediatric neuropathologists (S.A., A.K., V.P.C., W.P. M.H.) according to the WHO guidelines. Despite central review, we cannot exclude that a small number of samples are not PA but represent other low grade gliomas. Patients and tumor samples are described in Supplementary Table 1. A total of 280 patient tumors were included in our cohort, with copy number data available for 222 tumors, and $K I A A 1549-B R A F$ fusion/BRAF duplication data available for the majority of cases $(n=268)$. BRAF mutation status was available for 231 tumors and FGFR 1 hot spot mutation data available for 122 samples as described previously. A large cohort of PA tumors $(n=126)$ was utilized for gene expression profiling, assembling data from newly processed samples and previously published 
profiles of well-characterized tumor samples [9], using the Affymetrix HG-U133 plus 2.0 microarray (Affymetrix). Out of this gene expression cohort, all tumors $(n=$ 126) had age group information, $n=124$ had $B R A F$ fusion status, $n=108$ had $B R A F$ mutation status and $n$ $=122$ had available copy number information, with the techniques performed on individual samples described in Supplementary Table 1. Gene expression data was compared with other published datasets of glioblastoma $(n=84)$, pediatric high-grade glioma $(n=53)$, diffuseintrinsic pontine glioma (DIPG) $(n=37)$, ependymoma $(n=83)$ normal brain [54] and normal cerebellum [55] as controls. Age $\geq 18$ is classified as adult. Both adult and pediatric tumors were obtained from the London/Ontario Tumor Bank, and from collaborators in Canada, Hungary, the United Kingdom and Germany.

\section{RNA and DNA extraction}

RNA and DNA extraction methods for previously published cohorts are described in [6, 7, 9, 10, 15-17]. RNA and DNA were isolated from a subset of additional tumors using the Qiagen RNeasy Lipid Tissue Mini kit, Qiagen QIAmp/DNeasy DNA Mini kits respectively according to instructions of the manufacturer.

\section{KIAA1549-BRAF fusion screening}

$B R A F$ fusion status for previously described cohorts was determined as demonstrated in $[6,7,9,10,16,17]$. RT-PCR to detect the three most common KIAA1549$B R A F$ fusion transcripts was performed for a subset of additional samples as previously described [6].

\section{DNA copy number analysis}

For SNP analysis, DNA (250 ng) from PA samples was assayed with the HumanOmnil-Quad \& Human Omni2.5 genotyping beadchip platforms according to the recommendations of the manufacturer (Illumina, San Diego, CA, USA). For a subset of formalin-fixed paraffin-embedded (FFPE)-derived DNA samples ( $n=$ 13, Supplementary Table 1), DNA was first rescued with the Illumina FFPE restore assay kit (Illumina, Inc) and subsequently hybridized to the Illumina Omni2.5-8 v1.0 chip. These BeadChip platforms enable whole-genome genotyping of respectively over 1,140,419 (Omni1-Quad) and 2,379,855 (Omni2.5) tagSNP markers derived from the 1,000 Genomes project, all three HapMap phases, and recently published studies. Image intensities were extracted using Illumina's BeadScan software. Data for each BeadChip were self-normalized using information contained within the array. Copy number data was also included from previously published datasets utilizing SNP arrays and array-based comparative genomic hybridization (aCGH) $[6,7,10,15,16]$ with new analyses incorporating whole chromosome copy number profiling from $450 \mathrm{~K}$ methylation arrays (Illumina) utilizing the $\mathrm{R}$ package 'conumee' (http://www.bioconductor.org/packages/ release/bioc/html/conumee.html) essentially as described [56] and applied to published data [17]. In total, whole chromosomal copy number data was combined to yield a dataset of 222 tumors with numerical chromosome information to assess ploidy status.

\section{Gene expression profiling and analysis}

A large dataset of PA tumors $(n=126)$ [9] was combined for gene expression profiling using the online R2: microarray analysis and visualization platform (http:// r2.amc.nl). Ploidy status information was available for the majority of tumors with Affymetrix HG-U133 plus 2.0 gene expression data $(n=122)$ and was compared on this basis and on the basis of other molecular and clinical variables including $B R A F$ fusion (data available for $n=124$ tumors), BRAF mutation ( $n=108$ tumors) and age group ( $n=126$ tumors) through differential expression analysis using ANOVA at a false discovery rate (FDR) cut-off of $<0.001$ (as are displayed in Figure 2c, Figure 3 and Figure 4). A list of 558 genes passing FDR criteria were kept and utilized for further analyses of the aneuploid phenotype. Gene Ontology (GO) analysis was performed for these 558 differentially expressed genes utilizing the $\mathrm{R} 2$ database tool and presented in Figure 3a. Gene expression of MDM2 and PLK2 was assessed using absolute correlation analysis with results displayed in Figure 3e. Based on its elevated expression in aneuploid PA tumors compared with other brain tumors and normal tissue, MDM2 was pursued for additional correlation analyses. We investigated genes correlating (absolute correlation) with the expression of MDM2 at a stringent false discovery rate (FDR) corrected criterion of $<0.001$, which resulted in 741 robust combinations used for further Gene Ontology (GO) analysis and chromosome mapping in the dataset of 122 PA tumors profiled for aneuploidy. In control glioma and normal brain datasets detailed above, absolute correlation with identical FDR $<0.001$ was calculated for genes correlating with $M D M 2$. Representative heatmaps using transformed Z-scores illustrating correlated signatures are displayed in Supplementary Figure 2a-2c and were generated utilizing the R2 database tool. Exonic level expression of exons $1 \mathrm{a}, 1 \mathrm{~b}$ and 2 of MDM2 from previously published RNAsequencing data [9] was assessed to determine ratios of promoter 1 (P1) and P2 usage in a subset of PA tumors with available data $(n=69)$. 


\section{MDM2 SNP 309 sanger sequencing}

Sanger sequencing of the MDM2 promoter SNP 309 was performed on 201 tumors using HotStart FastTaq KIT (Kapa) using forward primer: 5'-TGGTGAGGAGCAGGTACTGG-3' and reverse primer: 5'-CGGAACGTGTCTGAACTTGA-3' with the following PCR cycling conditions: 1 cycle of 1 minute at $96^{\circ} \mathrm{C}, 33$ cycles of 10 seconds at $96^{\circ} \mathrm{C}, 5$ seconds at $58^{\circ} \mathrm{C}$ and 1 second at $72^{\circ} \mathrm{C}$ followed by 1 cycle at $72^{\circ} \mathrm{C}$ for 30 seconds.

\section{Quantitative RT-PCR determination of MDM2 expression levels}

Quantitative determination of $M D M 2$ expression levels was performed utilizing the Ssofast Evagreen kit (BioRad) with standard conditions indicated by the manufacturer at an annealing temperature of $58^{\circ} \mathrm{C}$ on the Roche LightCycler 480 (Roche) in a subset of 22 PA samples. Cycle threshold $\left(\mathrm{C}_{\mathrm{t}}\right)$ values were normalized to $\beta$-actin $(A C T B)$ and a calibrator normal brain sample with wild-type (TT) MDM2 SNP 309 genotype using the $2^{-\Delta \Delta C t}$ method. The following primers were used:

5'-TCTCAAGCTCCGTGTTTGGTCAGT-3', $M D M 2$ forward

5'-ACCTTGCAACAGCTGCAGATGAAC-3', $M D M 2$ reverse

5'-GGCACCCAGCACAATGAAGATCAA-3', $\beta$-actin forward 5'TAGAAGCATTTGCGGTGGACGATGGA-3'. $\beta$-actin reverse

\section{Statistical analyses}

Contingency (Fisher's Exact Test), unpaired, two-tailed t-test comparisons and one-way ANOVA were performed utilizing GraphPad Prism to determine $P$-values. ANOVA, absolute correlation and multiple test correction using false discovery rate (FDR) and Gene Ontology (GO) calculations were performed within R2. Kaplan-Meier analyses were performed within GraphPad Prism for tumors with available data: aneuploidy $(n=$ 193), BRAF fusion ( $n=233), B R A F$ mutation $(n=196)$, brain location $(n=235)$.

\section{ACKNOWLEDGMENTS}

The authors are indebted to François Bacot and Daniel Vincent and staff at the McGill University and Genome Quebec Innovation Centre for excellent technical assistance.

\section{CONFLICTS OF INTEREST}

The authors declare that they have no conflict of interest.

\section{GRANT SUPPORT}

This work was supported by the Cole Foundation and was funded by the Canadian Institute for Health Research (CIHR) and by the PedBrain Tumor Project contributing to the International Cancer Genome Consortium, funded by German Cancer Aid (109252) and by the German Federal Ministry of Education and Research (BMBF, grant \#01KU1201A). A. Klekner was supported by Hungarian Brain Research Program - Grant No. TIA_13_NAP-A-V/3, and the János Bolyai Scholarship of the Hungarian Academy of Sciences. A.M.F. is the recipient of a studentship award from the CIHR and an Award from the CIHR-Systems Biology Training Program of McGill University. M.S., K.J. are recipients of fellowships from the Foundation of Stars and the McGill Integrated Cancer Research Training Program. S.L. and V.P.C. are supported by The Brain Tumour Charity (UK). N.J. is the recipient of a Chercheur Clinicien Senior Award from Fonds de Recherche en Santé du Québec.

\section{Accession numbers}

Gene expression data utilized herein can be accessed through the Gene Expression Omnibus (GEO) at the following accession numbers:

GSE7696

GSE26576

GSE19578

GSE11882

GSE3526

\section{REFERENCES}

1. Louis DN, Ohgaki H, Wiestler OD, Cavenee WK, Burger PC, Jouvet A, Scheithauer BW and Kleihues P. The 2007 WHO classification of tumours of the central nervous system. Acta neuropathologica. 2007; 114:97-109.

2. Kieran MW, Walker D, Frappaz D and Prados M. Brain tumors: from childhood through adolescence into adulthood. Journal of clinical oncology : official journal of the American Society of Clinical Oncology. 2010; 28:47834789.

3. Dolecek TA, Propp JM, Stroup NE and Kruchko C. CBTRUS statistical report: primary brain and central nervous system tumors diagnosed in the United States in 2005-2009. Neuro-oncology. 2012; 14 Suppl 5:v1-49.

4. Ostrom QT, Gittleman H, Liao P, Rouse C, Chen Y, Dowling J, Wolinsky Y, Kruchko C and Barnholtz-Sloan 
J. CBTRUS Statistical Report: Primary Brain and Central Nervous System Tumors Diagnosed in the United States in 2007-2011. Neuro-oncology. 2014; 16 Suppl 4:iv1-iv63.

5. Ostrom QT, Gittleman H, Farah P, Ondracek A, Chen Y, Wolinsky Y, Stroup NE, Kruchko C and Barnholtz-Sloan JS. CBTRUS statistical report: Primary brain and central nervous system tumors diagnosed in the United States in 2006-2010. Neuro-oncology. 2013; 15 Suppl 2:ii1-56.

6. Jones DT, Kocialkowski S, Liu L, Pearson DM, Backlund LM, Ichimura $\mathrm{K}$ and Collins VP. Tandem duplication producing a novel oncogenic BRAF fusion gene defines the majority of pilocytic astrocytomas. Cancer research. 2008; 68:8673-8677.

7. Jones DT, Kocialkowski S, Liu L, Pearson DM, Ichimura $\mathrm{K}$ and Collins VP. Oncogenic RAF1 rearrangement and a novel BRAF mutation as alternatives to KIAA1549:BRAF fusion in activating the MAPK pathway in pilocytic astrocytoma. Oncogene. 2009; 28:2119-2123.

8. Jones DT, Gronych J, Lichter P, Witt O and Pfister SM. MAPK pathway activation in pilocytic astrocytoma. Cellular and molecular life sciences : CMLS. 2012; 69:1799-1811.

9. Jones DT, Hutter B, Jager N, Korshunov A, Kool M, Warnatz HJ, Zichner T, Lambert SR, Ryzhova M, Quang DA, Fontebasso AM, Stutz AM, Hutter S, Zuckermann M, Sturm D, Gronych J, et al. Recurrent somatic alterations of FGFR1 and NTRK2 in pilocytic astrocytoma. Nature genetics. 2013; 45:927-932.

10. Cin H, Meyer C, Herr R, Janzarik WG, Lambert S, Jones DT, Jacob K, Benner A, Witt H, Remke M, Bender S, Falkenstein F, Van Anh TN, Olbrich H, von Deimling A, Pekrun A, et al. Oncogenic FAM131B-BRAF fusion resulting from $7 \mathrm{q} 34$ deletion comprises an alternative mechanism of MAPK pathway activation in pilocytic astrocytoma. Acta neuropathologica. 2011; 121:763-774.

11. Schindler G, Capper D, Meyer J, Janzarik W, Omran H, Herold-Mende C, Schmieder K, Wesseling P, Mawrin C, Hasselblatt M, Louis DN, Korshunov A, Pfister S, Hartmann C, Paulus W, Reifenberger G, et al. Analysis of BRAF V600E mutation in 1,320 nervous system tumors reveals high mutation frequencies in pleomorphic xanthoastrocytoma, ganglioglioma and extra-cerebellar pilocytic astrocytoma. Acta neuropathologica. 2011; 121:397-405.

12. Zhang J, Wu G, Miller CP, Tatevossian RG, Dalton JD, Tang B, Orisme W, Punchihewa C, Parker M, Qaddoumi I, Boop FA, Lu C, Kandoth C, Ding L, Lee R, Huether R, et al. Whole-genome sequencing identifies genetic alterations in pediatric low-grade gliomas. Nature genetics. 2013; 45:602-612.

13. Hasselblatt M, Riesmeier B, Lechtape B, Brentrup A, Stummer W, Albert FK, Sepehrnia A, Ebel H, Gerss J and Paulus W. BRAF-KIAA1549 fusion transcripts are less frequent in pilocytic astrocytomas diagnosed in adults. Neuropathology and applied neurobiology. 2011; 37:803-
806.

14. Brokinkel B, Peetz-Dienhart S, Ligges S, Brentrup A, Stummer W, Paulus W and Hasselblatt M. A comparative analysis of MAPK pathway hallmark alterations in pilocytic astrocytomas: age-related and mutually exclusive. [corrected]. Neuropathology and applied neurobiology. 2015; 41:258-261.

15. Jones DT, Ichimura K, Liu L, Pearson DM, Plant K and Collins VP. Genomic analysis of pilocytic astrocytomas at $0.97 \mathrm{Mb}$ resolution shows an increasing tendency toward chromosomal copy number change with age. Journal of neuropathology and experimental neurology. 2006; 65(11):1049-1058.

16. Jacob K, Albrecht S, Sollier C, Faury D, Sader E, Montpetit A, Serre D, Hauser P, Garami M, Bognar L, Hanzely Z, Montes JL, Atkinson J, Farmer JP, Bouffet E, Hawkins C, et al. Duplication of $7 q 34$ is specific to juvenile pilocytic astrocytomas and a hallmark of cerebellar and optic pathway tumours. British journal of cancer. 2009; 101:722733.

17. Lambert SR, Witt H, Hovestadt V, Zucknick M, Kool M, Pearson DM, Korshunov A, Ryzhova M, Ichimura K, Jabado N, Fontebasso AM, Lichter P, Pfister SM, Collins VP and Jones DT. Differential expression and methylation of brain developmental genes define location-specific subsets of pilocytic astrocytoma. Acta neuropathologica. 2013; 126:291-301.

18. Post SM, Quintas-Cardama A, Pant V, Iwakuma T, Hamir A, Jackson JG, Maccio DR, Bond GL, Johnson DG, Levine AJ and Lozano G. A high-frequency regulatory polymorphism in the p53 pathway accelerates tumor development. Cancer cell. 2010; 18:220-230.

19. Bond GL, Hu W, Bond EE, Robins H, Lutzker SG, Arva NC, Bargonetti J, Bartel F, Taubert H, Wuerl P, Onel K, Yip L, Hwang SJ, Strong LC, Lozano G and Levine AJ. A single nucleotide polymorphism in the MDM2 promoter attenuates the p53 tumor suppressor pathway and accelerates tumor formation in humans. Cell. 2004; 119:591-602.

20. Manfredi JJ. The Mdm2-p53 relationship evolves: Mdm2 swings both ways as an oncogene and a tumor suppressor. Genes \& development. 2010; 24:1580-1589.

21. Paugh BS, Qu C, Jones C, Liu Z, Adamowicz-Brice M, Zhang J, Bax DA, Coyle B, Barrow J, Hargrave D, Lowe J, Gajjar A, Zhao W, Broniscer A, Ellison DW, Grundy $\mathrm{RG}$, et al. Integrated molecular genetic profiling of pediatric high-grade gliomas reveals key differences with the adult disease. Journal of clinical oncology : official journal of the American Society of Clinical Oncology. 2010; 28:30613068.

22. Paugh BS, Broniscer A, Qu C, Miller CP, Zhang J, Tatevossian RG, Olson JM, Geyer JR, Chi SN, da Silva NS, Onar-Thomas A, Baker JN, Gajjar A, Ellison DW and Baker SJ. Genome-wide analyses identify recurrent amplifications of receptor tyrosine kinases and cell-cycle 
regulatory genes in diffuse intrinsic pontine glioma. Journal of clinical oncology : official journal of the American Society of Clinical Oncology. 2011; 29:3999-4006.

23. Johnson RA, Wright KD, Poppleton H, Mohankumar KM, Finkelstein D, Pounds SB, Rand V, Leary SE, White E, Eden C, Hogg T, Northcott P, Mack S, Neale G, Wang YD, Coyle B, et al. Cross-species genomics matches driver mutations and cell compartments to model ependymoma. Nature. 2010; 466:632-636.

24. Theeler BJ, Ellezam B, Sadighi ZS, Mehta V, Tran MD, Adesina AM, Bruner JM and Puduvalli VK. Adult pilocytic astrocytomas: clinical features and molecular analysis. Neuro-oncology. 2014; 16:841-847.

25. Maurici D, Perez-Atayde A, Grier HE, Baldini N, Serra $\mathrm{M}$ and Fletcher JA. Frequency and implications of chromosome 8 and 12 gains in Ewing sarcoma. Cancer genetics and cytogenetics. 1998; 100:106-110.

26. Qi H, Dal Cin P, Hernandez JM, Garcia JL, Sciot R, Fletcher C, Van Eyken P, De Wever I and Van den Berghe H. Trisomies 8 and 20 in desmoid tumors. Cancer genetics and cytogenetics. 1996; 92:147-149.

27. Faggioli F, Vijg J and Montagna C. Chromosomal aneuploidy in the aging brain. Mechanisms of ageing and development. 2011; 132:429-436.

28. Rehen SK, McConnell MJ, Kaushal D, Kingsbury MA, Yang AH and Chun J. Chromosomal variation in neurons of the developing and adult mammalian nervous system. Proceedings of the National Academy of Sciences of the United States of America. 2001; 98:13361-13366.

29. Kaushal D, Contos JJ, Treuner K, Yang AH, Kingsbury MA, Rehen SK, McConnell MJ, Okabe M, Barlow C and Chun J. Alteration of gene expression by chromosome loss in the postnatal mouse brain. The Journal of neuroscience : the official journal of the Society for Neuroscience. 2003; 23:5599-5606.

30. Rehen SK, Yung YC, McCreight MP, Kaushal D, Yang AH, Almeida BS, Kingsbury MA, Cabral KM, McConnell MJ, Anliker B, Fontanoz M and Chun J. Constitutional aneuploidy in the normal human brain. The Journal of neuroscience : the official journal of the Society for Neuroscience. 2005; 25:2176-2180.

31. Faggioli F, Wang T, Vijg J and Montagna C. Chromosomespecific accumulation of aneuploidy in the aging mouse brain. Human molecular genetics. 2012; 21:5246-5253.

32. Kaul A, Chen YH, Emnett RJ, Dahiya S and Gutmann DH. Pediatric glioma-associated KIAA1549:BRAF expression regulates neuroglial cell growth in a cell type-specific and mTOR-dependent manner. Genes \& development. 2012; 26:2561-2566.

33. Kaul A, Chen YH, Emnett RJ, Gianino SM and Gutmann DH. Conditional KIAA1549:BRAF mice reveal brain region- and cell type-specific effects. Genesis. 2013; 51:708-716.

34. Cui $\mathrm{Y}$ and Guadagno TM. B-Raf(V600E) signaling deregulates the mitotic spindle checkpoint through stabilizing Mps1 levels in melanoma cells. Oncogene. 2008; 27:3122-3133.

35. Cui Y, Borysova MK, Johnson JO and Guadagno TM. Oncogenic B-Raf(V600E) induces spindle abnormalities, supernumerary centrosomes, and aneuploidy in human melanocytic cells. Cancer research. 2010; 70:675-684.

36. Liu J, Cheng X, Zhang Y, Li S, Cui H, Zhang L, Shi R, Zhao Z, He C, Wang C, Zhao H, Zhang C, Fisk HA, Guadagno TM and Cui Y. Phosphorylation of Mps1 by BRAFV600E prevents Mps1 degradation and contributes to chromosome instability in melanoma. Oncogene. 2013; 32:713-723.

37. Weaver BA, Silk AD, Montagna C, Verdier-Pinard P and Cleveland DW. Aneuploidy acts both oncogenically and as a tumor suppressor. Cancer cell. 2007; 11:25-36.

38. Oromendia AB, Dodgson SE and Amon A. Aneuploidy causes proteotoxic stress in yeast. Genes \& development. 2012; 26:2696-2708.

39. Chen G, Bradford WD, Seidel CW and Li R. Hsp90 stress potentiates rapid cellular adaptation through induction of aneuploidy. Nature. 2012; 482:246-250.

40. Gordon DJ, Resio B and Pellman D. Causes and consequences of aneuploidy in cancer. Nature reviews Genetics. 2012; 13:189-203.

41. Kang YH, Park JE, Yu LR, Soung NK, Yun SM, Bang JK, Seong YS, Yu H, Garfield S, Veenstra TD and Lee KS. Self-regulated Plk1 recruitment to kinetochores by the Plk1-PBIP1 interaction is critical for proper chromosome segregation. Molecular cell. 2006; 24:409-422.

42. Krause A and Hoffmann I. Polo-like kinase 2-dependent phosphorylation of NPM/B23 on serine 4 triggers centriole duplication. PloS one. 2010; 5:e9849.

43. Brevini TA, Pennarossa G, Maffei S, Tettamanti G, Vanelli A, Isaac S, Eden A, Ledda S, de Eguileor M and Gandolfi F. Centrosome amplification and chromosomal instability in human and animal parthenogenetic cell lines. Stem cell reviews. 2012; 8:1076-1087.

44. Cizmecioglu O, Krause A, Bahtz R, Ehret L, Malek N and Hoffmann I. Plk2 regulates centriole duplication through phosphorylation-mediated degradation of Fbxw7 (human Cdc4). Journal of cell science. 2012; 125:981-992.

45. Cizmecioglu O, Warnke S, Arnold M, Duensing S and Hoffmann I. Plk2 regulated centriole duplication is dependent on its localization to the centrioles and a functional polo-box domain. Cell cycle. 2008; 7:3548-3555.

46. Chang J, Cizmecioglu O, Hoffmann I and Rhee K. PLK2 phosphorylation is critical for CPAP function in procentriole formation during the centrosome cycle. The EMBO journal. 2010; 29:2395-2406.

47. Warnke S, Kemmler S, Hames RS, Tsai HL, HoffmannRohrer U, Fry AM and Hoffmann I. Polo-like kinase-2 is required for centriole duplication in mammalian cells. Current biology : CB. 2004; 14:1200-1207. 
48. Wang P, Lushnikova T, Odvody J, Greiner TC, Jones $\mathrm{SN}$ and Eischen CM. Elevated Mdm2 expression induces chromosomal instability and confers a survival and growth advantage to B cells. Oncogene. 2008; 27:1590-1598.

49. Lushnikova T, Bouska A, Odvody J, Dupont WD and Eischen CM. Aging mice have increased chromosome instability that is exacerbated by elevated Mdm2 expression. Oncogene. 2011; 30:4622-4631.

50. Marine JC, Francoz S, Maetens M, Wahl G, Toledo $\mathrm{F}$ and Lozano G. Keeping p53 in check: essential and synergistic functions of Mdm2 and Mdm4. Cell death and differentiation. 2006; 13:927-934.

51. Proctor CJ and Gray DA. Explaining oscillations and variability in the $\mathrm{p} 53-\mathrm{Mdm} 2$ system. BMC systems biology. 2008; $2: 75$.

52. Thompson SL and Compton DA. Proliferation of aneuploid human cells is limited by a p53-dependent mechanism. The Journal of cell biology. 2010; 188:369-381.

53. Valenti F, Fausti F, Biagioni F, Shay T, Fontemaggi G, Domany E, Yaffe MB, Strano S, Blandino G and Di Agostino S. Mutant p53 oncogenic functions are sustained by Plk2 kinase through an autoregulatory feedback loop. Cell cycle. 2011; 10:4330-4340.

54. Berchtold NC, Cribbs DH, Coleman PD, Rogers J, Head E, Kim R, Beach T, Miller C, Troncoso J, Trojanowski JQ, Zielke HR and Cotman CW. Gene expression changes in the course of normal brain aging are sexually dimorphic. Proceedings of the National Academy of Sciences of the United States of America. 2008; 105:15605-15610.

55. Roth RB, Hevezi P, Lee J, Willhite D, Lechner SM, Foster AC and Zlotnik A. Gene expression analyses reveal molecular relationships among 20 regions of the human CNS. Neurogenetics. 2006; 7:67-80.

56. Sturm D, Witt H, Hovestadt V, Khuong-Quang DA, Jones DT, Konermann C, Pfaff E, Tonjes M, Sill M, Bender S, Kool M, Zapatka M, Becker N, Zucknick M, Hielscher T, Liu XY, et al. Hotspot mutations in H3F3A and IDH1 define distinct epigenetic and biological subgroups of glioblastoma. Cancer cell. 2012; 22:425-437. 